\title{
ARTICLE
}

\section{Hepatitis C: a patient's journey from a psychiatrist's perspective}

\author{
Vellingiri Raja Badrakalimuthu, Daphne Rumball \& Amit Chawla
}

\begin{abstract}
Vellingiri Raja Badrakalimuthu is a Clinical Leadership Fellow and an honorary specialty registrar in old age liaison psychiatry in Cambridge, UK. His special interests include the neuroscience of old age and the interface between mental health and physical health. Daphne Rumball has been a consultant in substance misuse in Great Yarmouth since the setting up of the service in 1986. She is actively involved in research into service delivery and has published widely on addictions. Amit Chawla is a staff grade psychiatrist in substance misuse in Great Yarmouth. His special interests include the physical health of the substance-misusing population. Correspondence Dr V. R. Badrakalimuthu, Beechcroft, Fulbourn Hospital, Cambridge CB21 5EF, UK. Email: raja. badrakalimuthu@cpft.nhs.uk
\end{abstract}

\begin{abstract}
SUMMARY
There is an increasing prevalence of hepatitis $C$, particularly among intravenous drug users. Estimates of infection with the hepatitis $\mathrm{C}$ virus (HCV) in patients with severe mental illness in the USA have ranged from $8.5 \%$ to $19.6 \%$. Alcohol use and misuse is the single biggest contributory factor to the development of fatal liver disease in people with hepatitis C. Neuropsychiatric symptoms are present in HCV infection, independent of any treatment. Despite such alarming observations, the success rate of antiviral treatment in chronic hepatitis $C$ has improved considerably in recent years. This article explores psychiatrists' involvement in advocating for treatment inclusion, assessing patients' capacity to make treatment-related decisions and supporting the medical team in managing the complex journey a patient undertakes from being at high risk to receiving treatment for neuropsychiatric symptoms.

\section{DECLARATION OF INTEREST}

None.
\end{abstract}

It is estimated that between $0.4 \%$ and $1 \%$ of the UK population are infected with the hepatitis $\mathrm{C}$ virus (HCV), equating to between 250000 and 600000 individuals (Royal College of General Practitioners 2007). Around 50\% of intravenous drug users have been infected with the virus (Health Protection Agency 2010). The quality of life impairment in people with HCV infection is greater than in people with other liver diseases. It was not until the late 1990s that an association between severe mental illness and HCV was explored. The high rate of co-occurring substance use disorders and severe mental illness, coupled with HCV infection, warrants special consideration.

\section{Risk factors for HCV infection}

The most important factor for HCV infection is current or past injection drug use (Lauer 2001). The Health Protection Agency (2010) reported an increase in the prevalence of hepatitis $\mathrm{C}$ among those injecting drugs from $38 \%$ in 2000 to $47 \%$ in 2009 , with injecting drug use contributing to $90 \%$ of the infections. Only about a half of those who were hepatitis $\mathrm{C}$ sero-positive reported being aware of their positive status. The report also noted an increase in the incidence of hepatitis $\mathrm{C}$ among new injectors. In London, an estimated 86\% of injecting drug users had hepatitis C (Best 1999) - a higher incidence of HCV infection than in many cities worldwide (Judd 2005).

In the USA, estimates of HCV infection in cohorts of patients with severe mental illness have ranged from $8.5 \%$ to $19.6 \%$ (Rosenberg 2001; Dinwiddie 2003). One US study reported that $10.5 \%$ of inpatients with psychosis were positive for hepatitis C (Al-Jurdi 2003). Other studies from the USA found that $20.3 \%$ of long-term psychiatric in-patients and $9.9 \%$ of in-patients with schizophrenia were positive for HCV (Meyer 2003; Huckans 2006). The Epidemiologic Catchment Area population survey (Regier 1990) found that just under 1 in 3 people (29\%) with a mental disorder had a lifetime experience of a substance use disorder $(22 \%$ for alcohol and 15\% for drugs), just over 1 in 3 (37\%) of those with an alcohol use disorder had a lifetime experience of a mental health disorder, and 1 in 2 (53\%) of those with a drug use disorder had experienced a mental health disorder.

Possible explanations for the rising incidence in hepatitis $\mathrm{C}$ infection in the UK include changes in patterns of injecting drug use, with increasing use of crack cocaine, increases in the size of the population of injecting drug users over and above any protective interventions, and fewer than 1 in 4 being in drug treatment at any one time (Hickman 2004). The use of crack cocaine leads to high-risk injecting behaviour and risky sexual behaviour, providing a nexus of risk of blood-borne virus transmission and infections. In a US study of 276 people who had ever smoked or snorted cocaine, $4.7 \%$ were infected with HCV (Koblin 2003).

In their study involving inmates of eight prisons in England and Wales, Weild et al (2000) reported that $31 \%$ of the injecting drug users had anti-HCV antibodies.

Additional risk factors for infection are shown in Box 1. Alcohol use and misuse are the single biggest contributory factor to the development of fatal liver disease in those with hepatitis C: alcohol use synergistically accelerates liver damage in the presence of chronic HCV infection. 


\section{BOX 1 Risk factors for acquiring HCV infection}

- Injection drug use

- Substance-misuse-related risk behaviour (such as snorting)

- Multiple sexual partners/sexual promiscuity

- Low socioeconomic status

- Transfusions and transplantations

- Haemodialysis

- Household and occupational exposure

- Tattooing

- Prisons

(Adapted from Alter 1997)

\section{The hepatitis $\mathbf{C}$ virus and alcohol}

According to one US study, $46 \%$ of patients with alcoholic liver disease test positive for antibodies to HCV (Marsano 1998). Alcohol dependence without liver disease shows a prevalence of $4.8 \%$ for $\mathrm{HCV}$ (Corrao 1992). Heavy alcohol consumption worsens hepatic damage and accelerates progression of HCV infection (Schiff 1997). As harmful or dependent use of alcohol are related to HCV infection, general practitioners and psychiatrists should utilise consultation opportunities to raise awareness of blood-borne viruses, including HCV, to encourage testing for such viruses and to discuss preventive or therapeutic measures if appropriate. The British Society of Gastroenterology (Booth 2001) recommends that patients be advised to consume no more than $50 \mathrm{~g}$ (about 6 units) of alcohol per day. Further guidance on managing alcohol misuse and dependence in people with alcohol-mediated health problems is available from the Scottish Intercollegiate Guidelines Network (SIGN), the National Institute for Health and Clinical Excellence (NICE; National Collaborating Centre for Mental Health 2011) and the Substance Misuse Management in General Practice (SMMGP) network (Scottish Intercollegiate Guidelines Network 2003; RCGP Substance Misuse Unit 2007).

\section{The course of infection}

Hepatitis C is a single-stranded RNA virus of the Flaviviridae family, with at least six genotypes. Genotypes $1 \mathrm{a}$ or $1 \mathrm{~b}$ account for $70-80 \%$ of cases in the USA and Europe. In the UK, $50 \%$ of all HCV infections are by genotype 1 , which is less responsive to treatment. A higher percentage of genotype 3 compared with other genotypes is seen among intravenous drug users in England (Thomson 2005). Of those infected with genotype 3 , around
$20 \%$ develop acute hepatitis, $85 \%$ chronic hepatitis, 20-30\% cirrhosis/advanced liver disease, and a small percentage develop hepatocellular carcinoma.

The incubation period of an acute HCV infection is usually 6-9 weeks, during which time most people show no symptoms. In most cases, antiHCV antibodies develop by 3 months. The rate of progression of the disease is slow and variable, over $20-50$ years. About $25 \%$ of infected people clear the virus following acute infection, 20\% develop acute hepatitis and $20-30 \%$ of develop advanced liver disease or cirrhosis within 20 years, with a small percentage developing hepatocellular carcinoma.

A summary of key points about HCV illness is given in Box 2.

\section{HCV testing}

Diagnosing HCV infection provides an opportunity to give individuals invaluable information on life changes to slow the rate of $\mathrm{HCV}$ disease progression and on the availability of treatment, thus reducing individual morbidity and mortality. It also allows clinicians to give targeted advice on preventive measures to curb the spread of the infection to others, thus reducing population morbidity and mortality. Because of the implications of test results and the challenges of treatment, psychiatrists should be well informed about HCV and actively involved in advising their patients about the benefits and risks of testing.

National Institute for Health and Clinical Excellence (2009) guidelines recommend offering hepatitis screening for patients in methadone treatment, whereas the Royal College of General Practitioners (RCGP Substance Misuse Unit 2007) and SIGN (Scottish Intercollegiate Guidelines Network 2003) recommend offering screening to all people in high-risk groups. This will include all drug users who have ever injected and their partners. Although we understand that it is contentious, given the observed prevalence of $\mathrm{HCV}$ infection in psychiatric patients admitted with serious mental illness in the USA, we recommend

\section{BOX 2 HCV illness}

- HCV genotypes 1a and $1 \mathrm{~b}$ account for $70-80 \%$ of cases in the USA and Europe

- About 20\% develop acute hepatitis; about 20-30\% develop advanced liver disease or cirrhosis within 20 years; a small percentage develop hepatocellular carcinoma

- The most important risk factor for HCV infection is current or past injection drug use, with greatest risk of infection in the first year of injecting 
that routine screening be offered to these patients as well. Alcohol, drug and sexual history-taking is generally poor and therefore it would be difficult to identify reliably those who would be advised to have HCV screening. Offering screening to all patients with serious mental illness would ensure that all those at risk have the choice of opting out if their individual risk is then assessed to be low. This is the practice within most alcohol and drugs services (including our own) that treat a population with high prevalence of HCV, and such an offer of testing for hepatitis $\mathrm{C}$ could be extended across adult mental health services.

Screening for hepatitis C includes enzyme immunoassay for the HCV antibody. Quantitative HCV RNA assay by polymerase chain reaction (PCR) determines viraemia and is used to monitor treatment response. HCV genotyping can provide a guide to treatment and prognosis. Although a negative anti-HCV test generally excludes HCV infection, false-negative testing is seen in immunocompromised individuals and patients with acute infection of less than 3 months' duration. Antibody testing may need to be repeated if the exposure risk continues or the 3-month 'window' applies to a negative test result. Once HCV infection is confirmed, liver biopsy can provide information on the extent of liver disease, but it is not routinely required for access to treatment.

Any opportunity to test for HCV infection in atrisk populations should also be used to encourage testing for other blood-borne viruses such as hepatitis B and HIV. Patients should always be offered pre- and post-test counselling, along with encouragement for vaccination against hepatitis $\mathrm{B}$ and $\mathrm{A}$ as appropriate. Dried blood spot testing can be offered, especially to individuals who have difficult venous access due to damage from intravenous drug use (Hickman 2008).

\section{HCV diagnosis from a psychiatrist's perspective}

Given the observed prevalence of HCV infection in patients admitted with serious mental illness to a US psychiatric hospital, Dinwiddie et al (2003) recommend routine screening as a reasonable option in this population. Such testing, which should target people with risk factors for acquiring $\mathrm{HCV}$, should be accompanied by pre- and post-test counselling, as a diagnosis of HCV infection can have a very negative impact on pre-existing depression.

The presence of HCV is known to be associated with increased prevalence of depression before diagnosis, suggesting a central effect on mood in addition to the more general systemic effects of the infection. Therefore the role of the psychiatrist may include involvement in the decision about whether or not to proceed with the test. There may be issues of capacity and competence to consent to testing, as well as possible risk of exacerbation of psychiatric illness amounting even to suicidal behaviour in the event of an HCV-positive result. Rarely, there may be risks to third parties blamed for the transmission, either actually or delusionally. Such reactions cannot usually be addressed with standard counselling. Thus, a psychiatrist may prove to be an invaluable companion in a patient's journey even before a positive diagnosis is made.

\section{Treatment for HCV infection}

The success rate of antiviral treatment in chronic hepatitis $\mathrm{C}$ has improved considerably in recent years (Hadziyannis 2004; Zeuzem 2004). Despite this, the majority of clinical trials performed on combination therapy have omitted the largest single identifiable group of those infected with HCV intravenous drug users.

Treatment outcome and length of treatment required are linked most strongly to viral genotype, with genotype 1 having the least favourable prognosis. Estimation of the viral load is also relevant to prognosis. Treatment of HCV infection with pegylated interferon and ribavirin results in clearance of the virus in up to $50 \%$ of patients (Fried 2002), with those infected with genotypes 2 and 3 being three times more likely to achieve viral clearance. Genotypes 2 and 3 require treatment for 24 weeks, whereas genotype 1 requires treatment for 48 weeks. Genotype is therefore an important parameter to consider when advising on the risks and tolerability of treatment.

Nearly 50\% of the patients undergoing liver transplantation have end-stage liver disease due to HCV infection (Mueller 1994). The primary goals of transplantation are not only to ensure biological survival, but also to restore an optimal quality of life post-transplantation. The prevalence of psychiatric complications in this population is likely to be high.

\section{HCV treatment and drug treatment programmes}

Evidence has established that former and current intravenous drug users, including those on oral or injectable substitute maintenance therapy, need not be excluded from HCV therapy. Studies have shown that many patients, including those with continuing injecting drug use, can tolerate interferon therapy without undue worsening of their psychiatric or substance use disorders (Pariante 2002; Schaefer 2005). Studies of drug users who continue to inject after successful HCV treatment show that the risk of hepatitis $\mathrm{C}$ reinfection (e.g. because of return 
to unsafe injecting) in the period after sustained virological response is low (Matthews 2005).

The NICE guidelines on combination therapy (peginterferon alpha plus ribavirin) for continuing intravenous drug users with hepatitis $\mathrm{C}$ suggest that where treatment adherence is achieved, success rates are not significantly different from those for individuals who have stopped using intravenous drugs (National Institute for Clinical Excellence 2004). This welcome change is against the counterproductive traditional position of denying treatment to ongoing injecting drug users because of a presumed probability of reinfection, presumed likelihood of relatively high levels of non-adherence to treatment, and the possibility of drug interactions (Booth 2001). In practice, these theoretical risks do not adversely affect treatment outcome for those able to engage in the demanding treatment regime.

The SIGN guidelines (Scottish Intercollegiate Guidelines Network 2006) recommend that HCVpositive patients on a drug treatment programme be considered for treatment with a combination of pegylated interferon and ribavirin. Other suggestions include addressing general healthcare needs, drug-related harm reduction, comprehensive assessment of psychological needs and assessment of likeliness to adhere to antiviral treatment.

The US National Institutes of Health Consensus Development Program (2002) states that methadone treatment reduces risky behaviours that can spread HCV infection. It also notes that HCV therapy can be successful even when patients do not abstain from continued drug or alcohol use or are on daily methadone. European and US studies show that patients with hepatitis $\mathrm{C}$ infection receiving methadone maintenance treatment do persist with HCV therapy and do as well as other patients, bolstering the case for drug services to encourage clients to consider diagnostic testing and therapy for HCV (Novick 2008). Co-infection with HIV complicates treatment of both diseases, but in suitable patients antiviral therapy for HCV infection can still be effective. As already mentioned, many $\mathrm{HCV}$ patients receiving interferon experience no undue worsening of their psychiatric or substance use disorders (Schaefer 2005).

It should also be remembered that methadone maintenance treatment can have a positive effect on engagement in treatment for HCV infection and can help improve psychiatric symptoms and prognosis.

Key points about diagnosis and treatment are listed in Box 3.

\section{Preventing HCV infection among drug users}

The National Institute for Health and Clinical Excellence (2009) recommends that needle and
BOX 3 Diagnostic testing and treatment
- National and international guidelines recommend offering an $\mathrm{HCV}$ test to individuals at high risk of infection and who might benefit from knowing their HCV status

- Fifty percent of all UK infections are by genotype 1 , which is less responsive to antiviral treatment

- In addition to biological investigations, there should be robust assessment of psychosocial factors that can affect disease progression and access and adherence to treatment
- General measures in HCV treatment include ensuring continued adherence to substance misuse treatment, lifestyle modifications and hepatitis B immunisation

- The success rate of antiviral treatment in chronic hepatitis $\mathrm{C}$ has increased considerably in recent years, owing to interferon and ribavirin combination therapy

- NICE does not exclude injecting drug users from continuing with monotherapy or combination therapy syringe programmes, which form part of harm reduction measures among injecting drug users, should provide access to testing for blood-borne viruses, vaccination and referral for treatment. Expanding on this, NICE recommends that the harm minimisation strategy for injecting drug users should include access to opioid substitution therapy, opportunistic vaccination for hepatitis A, hepatitis B and tetanus, access to primary care services for general health advice and access to secondary care for treatment of hepatitis $\mathrm{C}$. There is good evidence for a direct effect of needle and syringe programmes on reducing the prevalence of hepatitis C (Wright 2006). These programmes provide a gateway for recruiting intravenous drug users to appropriate treatment programmes. There is also evidence to suggest that opiate substitution treatment programmes have some effect in reducing the incidence of HCV infection (Palmateer 2010).

\section{Neuropsychiatric effects across stages of HCV illness}

Neuropsychiatric symptoms occur in HCV infection, independent of any treatment (Zdilar 2000). Some are a direct effect of the infection, others result from asthenia and reduced quality of life. Different symptoms may occur at different stages of illness. Malaise, fatigue and depression have been reported during both acute and chronic stages of infection. Following the diagnosis of HCV infection, patients commonly experience a range of emotional reactions, including numbness, sadness, anger, blame, guilt, self-pity, anxiety and fear.

A comparative study of patients with HCV-related end-stage liver disease and patients with other liver diseases found that the HCV patients were younger and more vulnerable to depression (Singh 1997). The highest Beck Depression Inventory Scores were recorded in patients in the HCV group awaiting liver 
transplantation and not on interferon treatment. A significant proportion of such patients who do receive a transplant experience recurrent liver disease within the first year of transplantation and this stress can lead to relapse of depression. Other reasons for the higher rate of depression in HCV patients might be because, owing to their relatively young age, they lack adaptive mechanisms and feel greater loss of self-esteem.

In a study on emotional states and coping mechanisms, Kraus et al (2000) reported that people with recently diagnosed HCV infection showed the highest levels of problem-solving behaviour, and that depression scores were significantly higher 5 years after diagnosis than at the time of diagnosis.

In summary, although first receiving a diagnosis of HCV can be associated with psychological reaction, it is also associated with problem-solving behaviour. However, chronic illness and end-stage HCV-related liver disease are associated with significant depression.

\section{Neuropsychiatric adverse effects of IFN- $\alpha$}

Patients treated with interferon-alpha (IFN- $\alpha)$ most often experience flu-like symptoms, which remit within 1-2 weeks. Neurological adverse effects, including paraesthesia, delirium and extrapyramidal symptoms, have been reported in up to $33 \%$ of patients treated with the drug (Dusheiko 1997). Electroencephalograms (EEGs) may show generalised slow-wave activity, although there have been discordant reports (Suter 1984).

Neuropsychiatric side-effects are to be expected in the treatment of hepatitis $\mathrm{C}$ with IFN- $\alpha$, with prevalence rates reported in up to $70 \%$ of patients (Trask 2000). Interferon-alpha influences serotonergic, adrenergic and dopaminergic pathways through its effects on cytokines (interleukins and tumour necrosis factor-alpha, TNF- $\alpha$ ) and neuroendocrine systems, leading to manifestation of neuropsychiatric symptoms. Okanoue et al (1996) report that neuropsychiatric side-effects of treatment are more common in elderly patients. Serious neuropsychiatric effects require permanent discontinuation of interferon (Onyike 2004).

Fatigue is the most common and disabling sideeffect of interferon. Depressive symptoms, with a reported frequency up to $44 \%$, are a significant cause of premature discontinuation of interferon therapy (Crone 2004). Risk factors for interferoninduced depression, which begins to develop within the first 12 weeks of treatment, include high dose, longer duration of treatment and presence of preexisting subclinical symptoms (Hauser 2000; Musselman 2001). Diagnosis of depression is aided by structured and serial assessment using tools such as the Center for Epidemiologic Studies Depression
Scale (CES-D; Radloff 1977), which had been validated for use in people with chronic hepatitis $\mathrm{C}$.

Interferon-induced depression is considered to be a substance-induced mood disorder in which patients experience typical symptoms of depression. Suicidal intention has been recorded in many case reports. Increases in the levels of cytokines IL-6 and IL-8, reductions in serotonin and tryptophan, abnormal functioning of the hypothalamicpituitary-adrenal axis and reduced thyroxine have all been hypothesised to cause interferon-induced depression (Crone 2004). Hypometabolism in the bilateral dorsolateral prefrontal cortex observed on positron emission tomography scans of patients on interferon correlated with scores on the Beck Depression Inventory (Juengeling 2000).

Symptoms of anxiety develop in $10-20 \%$ of patients on interferon (Fontana 2000), although it is unclear whether they are independent of the depressive syndrome. An aetiology similar to that of depression has been hypothesised.

Interferon-induced mania and hypomania have been reported, and it is likely that there is also a risk of relapse of pre-existing psychotic illnesses, including schizophrenia (Crone 2004). We have seen this in our own clinical practice, and it sometimes requires discontinuation of therapy.

Cognitive side-effects suggestive of frontosubcortical impairment affecting motor coordination, psychomotor speed, verbal memory and executive function that improve on discontinuation of interferon have been documented. In patients who have progressed to hepatocellular carcinoma, IFN- $\alpha$ treatment is associated with significant cognitive impairment, including psychomotor slowing, perseveration, impaired visuospatial orientation and poor recall (Meyers 1992). The number of courses and duration of treatment have been correlated with the extent of neuropsychiatric symptoms (Amato 1995).

\section{Managing adverse effects of interferon}

Patient education, appropriate nutrition, rest, moderate exercise and restorative therapy may help patients cope with fatigue. Restorative therapy, comprising exercise and behavioural programmes, can address cognitive alterations and improve mood state, functional capacity and activity tolerance. Recombinant human erythropoietin, thyroid hormone supplements and psychostimulants have been used in clinical trials to treat it (Wagner 2000; Breitbart 2001).

Some studies support the prophylactic use of antidepressants for all patients receiving interferon (Musselman 2001; Dieperink 2003), although there are potential risks, such as retinal and gastrointestinal haemorrhage and secondary mania (Wienrieb 
2003). Decisions about early antidepressant use should take into account the patient's coping skills, support systems and life stressors, and patients should be educated about the risks and benefits of prophylactic antidepressant therapy. Selective serotonin reuptake inhibitors (SSRIs), especially fluoxetine and paroxetine, have been studied extensively in interferon-induced depression. Anorexia and fatigue responded less to paroxetine (Hauser 2000; Capuron 2002). Augmentation with psychostimulants (Loftis 2003) offers benefits in treating fatigue and cognitive dysfunction. However, the use of such treatments in individuals with a history of drug misuse should be very carefully considered. For treatment-refractory depression, electroconvulsive therapy might be an option (Bourgeois 2005). Cognitive-behavioural therapy, supportive psychotherapy and exercise have been reported to reduce IFN- $\alpha$-mediated depression.

Interferon-induced anxiety also responds to SSRIs. However, although it lacks specific trials in people with $\mathrm{HCV}$, the anticonvulsant pregabalin would be preferable, as it is excreted 98\% unchanged. It should be noted that, in high doses, pregabalin has been associated with exacerbation of underlying liver disease and therefore it should be prescribed with extreme caution. Gabapentin, another anticonvulsant that is not metabolised by the liver, may be an alternative choice (Greenberg 2000). Use of benzodiazepines for treating anxiety in a patient with a history of substance misuse requires caution owing to their addictive potential.

The management of mania or hypomania requires discontinuation of interferon, prompt psychiatric referral and initiation of mood stabilisers. Atypical antipsychotics are likely to be the first choice because of their ease of use, effectiveness and tolerability. Olanzapine-induced increased appetite and weight gain may counteract interferoninduced anorexia. In addition to its potential as an anxiolytic, gabapentin has mood-stabilisation and hypnotic properties. Lithium treatment may be more problematic because of the risk of hypothyroidism, which is also a risk of IFN- $\alpha$ treatment. Individuals with a history of intravenous drug use may have very poor venous access, making serum monitoring of lithium difficult.

Behavioural techniques used in early dementia, psychostimulants and naltrexone (Valentine 1995) can be useful in treating cognitive dysfunction. However, such interventions would be very unusual and naltrexone is contraindicated in the presence of opioid use.

Box 4 outlines guidelines for psychiatric management of interferon therapy. Comprehensive information on individual psychotropic drugs can be found in Crone et al (2006).
BOX 4 Suggested guidelines for psychiatric management of interferon therapy

- Baseline psychiatric evaluations prior to initiation of interferon therapy

- Patient information and education about the neuropsychiatric adverse effects and treatment options

- Treating significant baseline neuropsychiatric symptoms prior to initiation of interferon therapy

- For patients with a history of substance misuse, relapse prevention programmes should be initiated

- Patients at greater risk of developing neuropsychiatric symptoms (e.g. those with a history of depression) and those who develop such symptoms during interferon treatment should actively be managed by psychiatric services in collaboration with gastroenterologists

Key points about neuropsychiatric symptoms and their management are listed in Box 5.

\section{Service provision}

Studies have shown that outcomes for patients with alcohol- and drug-induced physical disorders are better when medical and addictions services work together than when the services operate separately (Samet 2001). The British Society of Gastroenterology (Booth 2001) recommends that all patients with HCV infection have access to appropriate diagnostic and therapeutic options, including referral to specialist clinician and counselling services. It also recommends regular psychological assessment of patients. These recommendations support the need for a robust pathway linking substance misuse psychiatry and gastroenterology/hepatology services. The psychiatrist's role in the patient's journey along this pathway is outlined in Fig. 1.

\section{B0X 5 Neuropsychiatric symptoms and their management}

- People with HCV-related liver disease are more vulnerable to depression than are people with other liver disorders

- Up to $44 \%$ of patients experience depressive symptoms during interferon therapy

- About $10-20 \%$ of patients on interferon develop anxiety symptoms

- SSRls, especially fluoxetine and paroxetine, have been studied extensively for treating interferon-induced depression

- Interferon-induced anxiety responds to SSRIs

- Atypical antipsychotics are likely to be the first choice in treatment of interferon-induced mania or psychosis 
One of us (D.R.) has a psychiatric liaison role with a gastroenterology service from within an alcohol and drugs service. The following pathway has been set up for patients with hepatitis C. The hepatology team assesses all patients using a guideline for eliciting mental health history. If the patient is being considered for antiviral treatment, this is followed by an in-depth assessment by the liaison hepatology nurse. This enables identification of individuals with current symptoms of suspected psychiatric illness. The liaison hepatology nurse, in consultation with psychiatrist, can then initiate biological and psychosocial interventions targeting these symptoms. The clinic then refers the patient to the consultant psychiatrist for further assessment in collaboration with the multidisciplinary team, and onward referral where appropriate. An alternate pathway might be to refer initially to a psychiatric nurse acting in a liaison role.

We advocate the setting up of a model that first capitalises on local interest and then refining it according to available resources. Whatever the

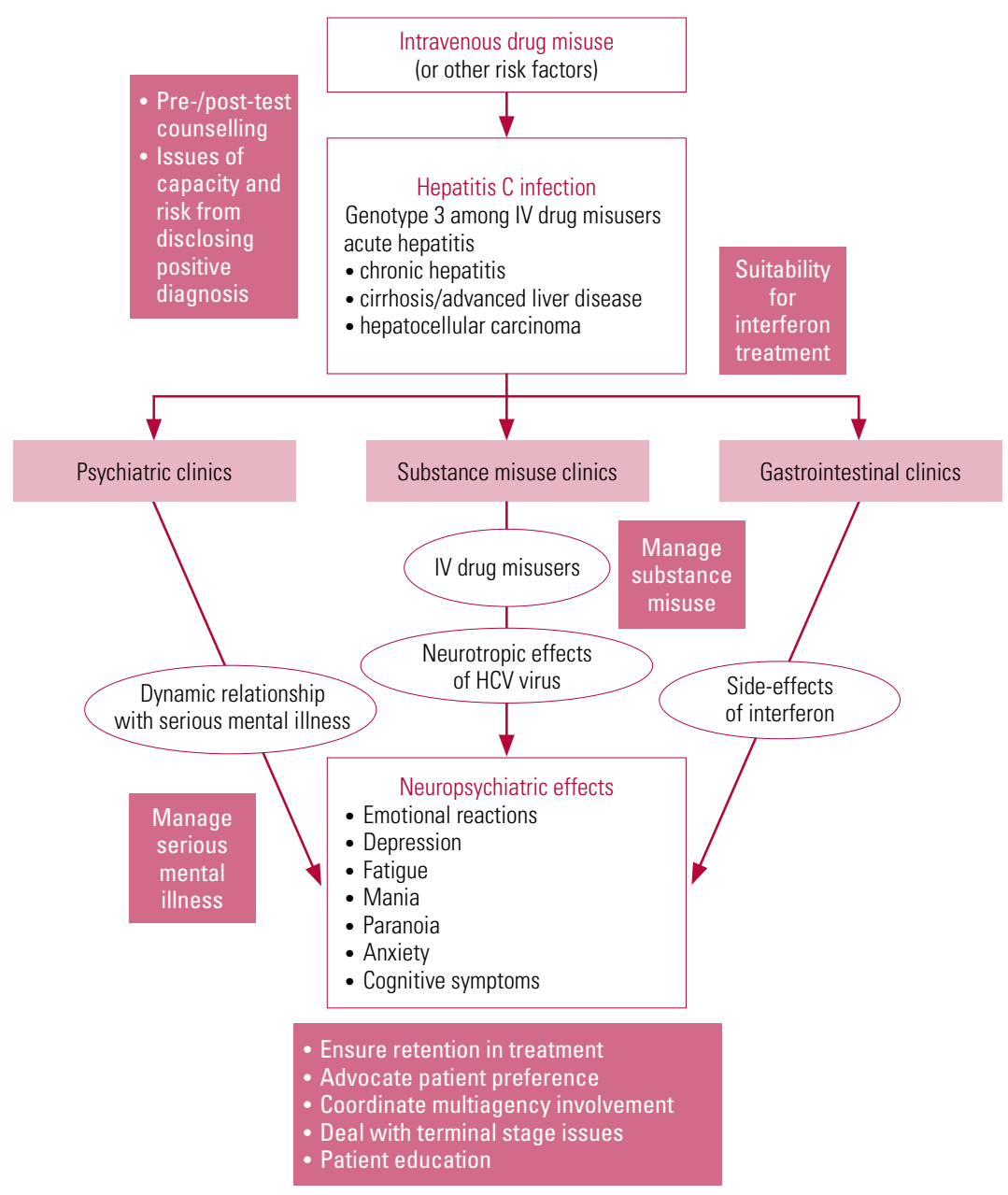

The psychiatrist's role in the journey of patients with hepatitis $\mathrm{C} . \mathrm{HCV}$, hepatitis $\mathrm{C}$ virus; IV, intravenous. chosen service provision, the essential issue is for the psychiatric assessment to be undertaken by someone with a good knowledge of the diagnostic and treatment issues outlined in this article and for that person to have referral rights to local psychiatric teams for further treatment where needed. It should be borne in mind that the course of HCV treatment is long and it would therefore not usually be appropriate for ongoing interventions to remain with the liaison psychiatry team.

The issue of how to set up collaborative working is, in our opinion, best worked out locally. Nevertheless, the key professionals must be involved and consultation must be a two-way process between hepatology and psychiatric services. Joint discussions should allow those involved to develop a pragmatic pathway that takes into consideration the difficulties that patients can face in accessing services. It can be very helpful to appoint lead nurses in each area (hepatology, drug and alcohol services, and psychiatry) to maintain good working arrangements. An in-reach approach can facilitate initial and ongoing engagement of patients at locations with which they are most familiar and where they have the support of their usual key workers. In-reach consultations also help patients who are experiencing fatigue to receive assessment and treatment by all relevant professions with a minimum of stress.

The common side-effects of combination treatment mimic opiate withdrawal and the use of syringes for interferon treatment may serve as a cue to trigger relapse of drug misuse. Psychiatric assessment by addictions specialists can help identify patients at risk of relapse and channel them through appropriate pharmacological and psychosocial interventions.

Substance misuse and psychiatric services can refer and enable attendance at HCV support groups that allow patients to help and educate one another about HCV infection, the steps involved in pretreatment evaluation and management of adverse effects during treatment.

\section{Training clinicians}

Inconsistent assessment and management by psychiatrists can lead to underreporting of neuropsychiatric symptoms resulting from HCV infection and treatment. A concerted effort is needed to train clinicians in psychiatric services, so that outcomes for patients with HCV infection are optimised (Box 6). A checklist such as that shown in Fig. 2 might help in discussing and documenting history on blood-borne viruses and treatment.

One of the primary goals in training a psychiatrist should be to enable them to address the stigma and 
common misconceptions of the patient and break down the barriers that prevent access to medical services.

The role of the psychiatrist in the treatment of hepatitis C is threefold (Freudenreich 2007):

1 to advocate for antiviral treatment if indicated;

2 to assess patients' capacity to make treatmentrelated decisions, their coping skills and the risks involved; and

3 to support the medical treatment team in multimodal assessment and treatment.

Rifai et al (2006) found that $21 \%$ of people with substance dependence referred to a gastroenterologist for suspected HCV infection did not attend the consultation. Clearly, much work needs to be done to determine how to engage patients more successfully. Unfortunately, the NICE guidelines on pegintereferon alpha for mild chronic hepatitis $\mathrm{C}$ recommend haematological assessment but not psychiatric assessment before treatment is started (National Institute for Clinical Excellence 2004). However, SIGN (Scottish Intercollegiate Guidelines Network 2003) and the British Society of Gastroenterology (Booth 2001) stress the value of psychiatric skills in the clinical care of patients with hepatitis $\mathrm{C}$ and advocate their inclusion in the care pathway. The inclusion of this substantial role has implications for training clinicians from hepatology and gastroenterology services and in service resources.

The cognitive effects of HCV infection, coupled with comorbid psychiatric illnesses and interferoninduced neuropsychiatric side-effects, necessitate the participation of psychiatrists in patients' care. During the initial treatment discussions, psychiatrists can be involved in determining that a patient is competent to agree to or refuse interferonbased treatment, with a full understanding of its risks and benefits. Motivational interviewing techniques

BOX 6 Focus of clinical training

- Increased awareness and clinical skills in assessment for early identification of neuropsychiatric symptoms

- Implementation of appropriate pharmacological and psychosocial interventions to treat such symptoms

- Use of motivational techniques to help patients to engage with and complete HCV treatments

- Ability to use structured scales through serial assessments to guide treatment and as a selfassessment exercise for the patient

- Working as an advocate for the patient and supporting the medical treatment team in multimodal assessment and treatment

\begin{tabular}{|c|c|c|}
\hline \multicolumn{3}{|c|}{ Blood-borne viruses assessment checklist } \\
\hline Patient's name & & Patient identifier \\
\hline Intravenous drug misuse? & Yes/No & \\
\hline \multicolumn{3}{|l|}{ Site(s) of injection } \\
\hline Sharing of needles? & Yes/No & \\
\hline $\begin{array}{l}\text { HCV } \\
\text { Not tested/Tested }\end{array}$ & (Result _ & When _ \\
\hline $\begin{array}{l}\text { HBV } \\
\text { Immunised/Not immunised } \\
\text { Not tested/Tested }\end{array}$ & (Result & When _l \\
\hline $\begin{array}{l}\text { HIV } \\
\text { Not tested/Tested }\end{array}$ & (Result _ & When _l \\
\hline $\begin{array}{l}\text { If not tested for BBV } \\
\text { Test offered? }\end{array}$ & Yes/No & \\
\hline \multicolumn{3}{|l|}{$\begin{array}{l}\text { If BBV test refused } \\
\text { Reasons: }\end{array}$} \\
\hline Treatment for BBV if positive & \multicolumn{2}{|c|}{ Seeking treatment/Not seeking treatment } \\
\hline \multicolumn{3}{|l|}{$\begin{array}{l}\text { If not seeking treatment } \\
\text { Reasons: }\end{array}$} \\
\hline \multicolumn{3}{|l|}{$\begin{array}{l}\text { If seeking treatment } \\
\text { Stage of treatment: }\end{array}$} \\
\hline \multicolumn{3}{|c|}{ Key professionals (with contact details) involved in BBV treatment: } \\
\hline BBV, blood-borne viruses; HBV, hep & tis B virus; HCV, hepat & s C virus \\
\hline
\end{tabular}

FIG 2

Blood-borne viruses assessment checklist.

can be particularly effective in this context. Hepatologists may be more willing to treat patients who have substance-related HCV if they know that the patients will receive close and continuous psychiatric monitoring. Rifai et al (2006) proffer a risk:benefit analysis that weighs two main factors: the likelihood of viral clearing and the likelihood of interferon-induced neuropsychiatric side-effects. Psychiatrists should be trained to assess other social and psychological variables, such as mode of acquisition and patients' coping mechanisms.

\section{Service implications}

As demonstrated in this review, there are many areas in which a collaborative care pathway involving hepatology and substance misuse services can be developed. These include encouraging injecting drug users with HCV infection to stay in treatment and assessing mental illness among those in treatment for HCV infection. The growing literature documents the neuropsychiatric complications of HCV infection and the necessity for psychiatric services to work collaboratively with medical services to produce best possible treatment outcomes. Psychiatrists should therefore develop extensive knowledge and clinical skills to assess and utilise appropriate resources to work together with the patient and medical services. 


\section{MCO answers}

1 b $\quad 2$ e $\quad 3 c \quad 4 b \quad 5 d$

\section{References}

Al-Jurdi RK, Burruss JW (2003) Prevalence of hepatitis C in psychiatric institutions. Psychosomatics 44: 439-40.

Alter JM (1997) Epidemiology of hepatitis C. Hepatology 26 (suppl 1): $625-55$.

Amato R, Meyers C, Ellerhorst J, et al (1995) A phase 1 trial of intermittent high-dose alpha interferon and dexamethasone in metastatic renal cell carcinoma. Annals of Oncology 6: 911-4.

Best D, Noble A, Finch F, et al (1999) Accuracy of perceptions of hepatitis $B$ and $C$ status: cross-sectional investigations of opiate addicts in treatment. BMJ 319: 290-1.

Booth JCL, O'Grady J, Neuberger J (2001) Clinical guidelines to the management of hepatitis C. Gut 49 (suppl 1): 1-21.

Bourgeois JA, Lorenzo R, Canning RD (2005) Depression as co-pilot: clinical implications of hepatitis C and interferon/ribavirin treatment. Psychiatric Times 22 (issue 5: April bonus edition): 1-7.

Breitbart W, Rosenfeld B, Kaim M, et al (2001) A randomized, doubleblind, placebo-controlled trial of psychostimulants for the treatment of fatigue in ambulatory patients with human immunodeficiency virus disease. Archives of Internal Medicine 161: 411-20.

Capuron L, Gumnick JF, Musselman DL, et al (2002) Neurobehavioural effects of interferon-alfa in cancer patients: phenomenology and paroxetine responsiveness of symptom dimensions. Neuropsychopharmacology 26: 643-52.

Corrao G, Carle F, Lepore AR, et al (1992) Interaction between alcohol consumption and positivity for antibodies to hepatitis $C$ virus on the risk of liver cirrhosis: a case-controlled study. European Journal of Epidemiology 8: 634-9.

Crone CC, Gabriel GM, Wise TN (2004) Managing the neuropsychiatric side-effects of interferon-based therapy for hepatitis C. Cleveland Clinical Journal of Medicine 71: S27-32.

Crone CC, Gabriel GM, DiMartini A (2006) An overview of psychiatric issues in liver disease for the consultation-liaison psychiatrist. Psychosomatics 47: 188-205

Dieperink E, Ho S, Thuras $P$, et al (2003) A prospective study of neuropsychiatric symptoms associated with interferon-alpha2b and ribavirin therapy for patients with chronic hepatitis C. Psychosomatics 44: 104-12.

Dinwiddie SH, Shicker L, Newman T (2003) Prevalence of hepatitis C among psychiatric patients in the public sector. American Journal of Psychiatry 160: 172-4.

Dusheiko G (1997) Side-effects of alpha-interferon in chronic hepatitis. Hepatology 26 (suppl 1): 112S-21S.

Fontana RJ (2000) Neuropsychiatric toxicity of antiviral treatment in chronic hepatitis C. Digestive Disorders 16: 107-16.

Freudenreich 0, Gandhi R, Walsh J, et al (2007) Hepatitis C in schizophrenia: screening experience in a community dwelling clozapine cohort. Psychosomatics 48: 405-11.

Fried MW, Shiffman ML, Reddy R, et al (2002) Peginterferon alfa-2a plus ribavirin for chronic hepatitis $\mathrm{C}$ virus infection. New England Journal of Medicine 347: 975-82.

Greenberg DB, Jonasch E, Gadd MA, et al (2000) Adjuvant therapy of melanoma with interferon-alpha-2 $b$ is associated with mania and bipolar syndromes: gabapentin may serve as a mood stabiliser. Cancer 89: 356-62.

Hadziyannis SJ, Sette H Jr, Morgan TR, et al (2004) Peginterferonalpha2a and ribavirin combination therapy in chronic hepatitis $\mathrm{C}$ : a randomized study of treatment duration and ribavirin dose. Annals of International Medicine 140: 346-55.

Hauser P, Soler R, Reed S, et al (2000) Prophylactic treatment of depression induced by interferon-alfa. Psychosomatics 41: 439-41.

Health Protection Agency (2010) Shooting Up: Infections among Injecting Drug Users in the UK 2009 (An Update: November 2010). Health Protection Agency (http://www.hpa.org.uk/web/HPAwebFile/ HPAweb C/1287143384395).
Hickman M, Higgins V, Hope VD, et al (2004) Injecting drug use in Brighton, Liverpool and London: best estimates of prevalence and coverage of public health indicators. Journal of Epidemiological Community Health 58: $766-71$.

Hickman M, McDonald T, Judd A, et al (2008) Increasing the uptake of hepatitis $C$ virus testing among injecting drug users in specialist drug treatment and prison settings by using dried blood spots for diagnostic testing: a cluster randomised trial. Journal of Viral Hepatitis 15: 250-4.

Huckans MS, Blackwell AD, Harms TA, et al (2006) Management of hepatitis $C$ disease among VA patients with schizophrenia and substance use disorders. Psychiatric Services 57: 403-6.

Judd A, Hickman M, Jones S, et al (2005) Incidence of hepatitis $C$ virus and HIV among new injecting drug users in London: prospective cohort study. BMJ 330: 24-5.

Juengeling FD, Ebert D, Gut 0, et al (2000) Prefrontal cortical hypometabolism during low-dose interferon alpha treatment. Psychopharmacology 152: 383-9.

Koblin BA, Factor SH, Wu Y, et al (2003) Hepatitis C virus infection among noninjecting drug users in New York City. Journal of Medical Virology 70: 387-90.

Kraus MR, Schafer A, Csef H, et al (2000) Emotional state, coping styles and somatic variables in patients with chronic hepatitis C. Psychosomatics 41: 377-84.

Lauer GM, Walker BD (2001) Hepatitis C virus infection. New England Journal of Medicine 345: 41-52

Loftis JM, Hauser $\mathrm{P}$ (2003) Comanagement of depression and HCV treatment. Psychiatric Annals 33: 385-91.

Marsano LS, Pena LR (1998) The interaction of alcoholic liver disease and hepatitis C. Hepatogastroenterology 45: 331-9.

Matthews G, Kronborg I, Dore G (2005) Treatment for hepatitis C virus infection among current injection drug users in Australia. Clinical Infectious Diseases 40: S325-9.

Meyer JM (2003) Prevalence of hepatitis A, hepatitis B and HIV among hepatitis C-seropositive state hospital patients: results from Oregon State Hospital. Journal of Clinical Psychiatry 64: 540-5.

Meyers CA, Abbruzzese JL (1992) Cognitive functioning in cancer patients: effects of previous treatment. Neurology 42: 434-6.

Mueller AR, Platz KP, Bechstein WO, et al (1994) Increased rejection after liver transplantation in FK 506-treated patients is associated with viral hepatitis C disease. Transplantation Procedures 26: 3637-9.

Musselman DL, Lawson DH, Gumnick JF, et al (2001) Paroxetine for the prevention of depression induced by high-dose interferon alfa. New England Journal of Medicine 344: 961-6.

National Collaborating Centre for Mental Health (2011) Alcohol-Use Disorders: Diagnosis, Assessment and Management of Harmful Drinking and Alcohol Dependence (NICE Clinical Guideline 115). National Institute for Health and Clinical Excellence (http://www.nice.org.uk/nicemedia/ live/13337/53191/53191.pdf)

National Institute for Clinical Excellence (2004) Interferon Alfa (Pegylated and Non-Pegylated) and Ribavirin for the Treatment of Chronic Hepatitis C (Technology Appraisal TA75). NICE (http://www.nice.org.uk/ nicemedia/pdf/TA075guidance.pdf).

National Institute for Health and Clinical Excellence (2009) Needle and Syringe Programmes: Providing People Who Inject Drugs with Injecting Equipment (NICE Public Health Guidance 18). NICE (http://www.emcdda. europa.eu/attachements.cfm/att_102215_EN_UK16_NSP.pdf).

National Institutes of Health Consensus Development Program (2002) Management of Hepatitis C: 2002 (National Institutes of Health Consensus Conference Statement). NIH (http://consensus.nih.gov/2002/ 2002HepatitisC2002116html.htm).

Novick DM, Kreek MJ (2008) Critical issues in the treatment of hepatitis $\mathrm{C}$ virus infection in methadone maintenance patients. Addiction 103: 905-18.

Okanoue T, Sakamoto S, Itoh Y, et al (1996) Side-effects of high-dose interferon therapy for chronic hepatitis C. Journal of Hepatology 25 . 283-91. 
Onyike CU, Bonner JO, Lykestos CG, et al (2004) Mania during treatment of chronic hepatitis $C$ with pegylated interferon and ribavirin. American Journal of Psychiatry 161: 429-35.

Palmateer N, Kimber J, Hickman M, et al (2010) Evidence for the effectiveness of sterile injecting equipment provision in preventing hepatitis $C$ and human immunodeficiency virus transmission among injecting drug users: a review of reviews. Addiction 105: 844-59.

Pariante CM, Landau S, Carpinello B (2002) Interferon alfa-induced adverse effects in patients with a psychiatric diagnosis. New England Journal of Medicine 347: 148-9.

Radloff LS (1977) The CES-D scale: a self report depression scale for research in the general population. Applied Psychological Measurement 1: 385-401.

RCGP Substance Misuse Unit, RCGP Sex, Drugs \& HIV Task Group, Substance Misuse Management in General Practice, et al (2007) Guidance for the Prevention, Testing, Treatment and Management of Hepatitis C in Primary Care. Royal College of General Practitioners (http:// www.smmgp.org.uk/download/guidance/guidance003.pdf).

Regier DA, Farmer ME, Rae DS, et al (1990) Co-morbidity of mental disorders with alcohol and other drug abuse: results from the Epidemiologic Catchment Area (ECA) study. JAMA 264: 2511-8.

Rifai MA, Moles JK, Lehman LP, et al (2006) Hepatitis C screening and treatment outcomes in patients with substance use/dependence disorders. Psychosomatics 47: 112-21.

Rosenberg SD, Goodman LA, Osher FC, et al (2001) Prevalence of HIV, hepatitis B and hepatitis $C$ in people with severe mental illness. American Journal of Public Health 91: 31-7.

Royal College of General Practitioners (2007) Guidance for the Prevention, Testing and Management of Hepatitis C in Primary Care. Royal College of General Practitioners.

Samet J, Freidmann P, Saitz R (2001) Benefits of linking primary medical care and substance abuse services: patient, provider and societal perspectives. Archives of Internal Medicine 161: 85-91.

Schaefer M, Schwaiger M, Garkisch AS, et al (2005) Prevention of interferon-alpha associated depression in psychiatric risk patients with chronic hepatitis C. Journal of Hepatology 42: 793-8.

Schiff ER (1997) Hepatitis C and alcohol. Hepatology 26 (suppl 1): 39S-42S.
Scottish Intercollegiate Guidelines Network (2003) The Management of Harmful Drinking and Alcohol Dependence in Primary Care (SIGN Guideline no. 74). SIGN.

Scottish Intercollegiate Guidelines Network (2006) Management of Hepatitis C: A National Clinical Guideline (SIGN Guideline no. 92). SIGN.

Singh N, Gayowski T, Wagener MM, et al (1997) Vulnerability to psychologic distress and depression in patients with end-stage liver disease due to hepatitis C virus. Clinical Transplantation 11: 406-11.

Suter CC, Westmoreland BF, Sharbrough FW, et al (1984) Electroencephalographic abnormalities in interferon encephalopathy: a preliminary report. Mayo Clinic Proceedings 59: 847-50.

Thomson BJ, Finch RG (2005) Hepatitis C virus infection. Clinical Microbiology of Infections 11: 86-94

Trask PC, Esper P, Riba M, et al (2000) Psychiatric side-effects of interferon therapy: prevalence, proposed mechanisms, and future directions. Journal of Clinical Oncology 18: 2316-26.

Valentine AD, Meyers CA, Talpaz M (1995) Treatment of neurotoxic sideeffects of interferon-alpha with naltrexone. Cancer Investigations 13 561-6.

Wagner GJ, Rabkin R (2000) Effects of dextroamphetamine on depression and fatigue in men with HIV: a double-blind, placebo-controlled trial. Journal of Clinical Psychiatry 61: 436-40.

Weild AR, Gill ON, Bennett SJM, et al (2000) Prevalence of HIV, hepatitis $B$, and hepatitis $C$ antibodies in prisoners in England and Wales: a national survey. Communicable Diseases and Public Health 3: 121-6.

Wienrieb RM, Auriacombe M, Lynch KG, et al (2003) A critical review of selective serotonin reuptake inhibitor-associated bleeding: balancing the risk of treating hepatitis-C infected patients. Journal of Clinical Psychiatry 64: 1502-10.

Wright N, Tompkins C (2006) A review of the evidence for the effectiveness of primary prevention interventions for hepatitis $C$ among injecting drug users. Harm Reduction Journal 3: 27

Zdilar D, Franco-Bronson K, Buchler N, et al (2000) Hepatitis C, interferon alfa, and depression. Hepatology 31: 1207-11.

Zeuzem S, Diago M, Gane E, et al (2004) Peginterferon alfa-2a (40 kilodaltons) and ribavirin in patients with chronic hepatitis $\mathrm{C}$ and normal aminotransferase level. Gastroenterology 127: 1724-32.

\section{MCOs}

Select the single best option for each question stem

1 As regards $\mathrm{HCV}$ infection:

a estimates of HCV infection in cohorts of patients with severe mental illness range from $1 \%$ to $5 \%$

b injecting drug use contributes to $90 \%$ of HCV infections

c in the UK, 50\% of all HCV infections are caused by genotype 2

d the prevalence of HCV in the UK population is estimated to be $0.1 \%$

e the prevalence of hepatitis $\mathrm{C}$ among injecting drug user has fallen from $47 \%$ in 2000 to $48 \%$ in 2009 .

2 The prevalence of anti-HCV antibodies among prisoners with a history of injecting drug use is about:

a $10 \%$

b $15 \%$ c $20 \%$

d $25 \%$

e $30 \%$.

3 As regards HCV and its treatment:

a genotype 3 is associated with good prognosis

b less than $25 \%$ report neuropsychiatric adverse effects from treatment

c patients with end-stage liver disease due to HCV infection comprise nearly $50 \%$ of the patients undergoing liver transplantation

d serious neuropsychiatric side-effects of treatment are more common in young patients

e treatment of HCV infection with pegylated interferon and ribavirin clears the virus in about $25 \%$ of patients.

\section{As regards neuropsychiatric adverse effects of interferon treatment:}

a decreased IL-6 levels are hypothesised to be associated with depression b fatigue is the most common symptom

c hypermetabolism is seen in the bilateral dorsolateral prefrontal cortex

d less than $5 \%$ of patients report symptoms of anxiety

e cognitive tests commonly show impairment of visual but not of verbal memory.

5 As regards treatment of neuropsychiatric adverse effects:

a interferon-induced anorexia responds well to paroxetine

b gabapentin should not be used to treat anxiety because it is metabolised in the liver

c naltrexone as a treatment for cognitive impairment is appropriate in the presence of opioid use

d retinal haemorrhage is a potential adverse effect of using a prophylactic antidepressant with interferon

e thyroid hormone supplements are ineffective in treating fatigue. 